Research Article

\title{
Terahertz Sensor Study Based on Spoof Surface Plasmon Polaritons
}

\author{
Ruiqi Zhao, ${ }^{1}$ Guizhen Lu ${ }^{D},{ }^{1}$ Hongcheng Yin, ${ }^{2}$ Jingjing Liang, ${ }^{1}$ Dongdong Zeng, \\ and Huaibao Xiao ${ }^{1}$
}

${ }^{1}$ College of Electronic Engineering, Communication University of China, Beijing 100024, China

${ }^{2}$ Beijing Institute of Environmental Laboratory, Beijing 100039, China

Correspondence should be addressed to Guizhen Lu; luguizhen@cuc.edu.cn

Received 13 August 2020; Revised 24 September 2020; Accepted 24 October 2020; Published 12 November 2020

Academic Editor: Mauro Parise

Copyright ( 92020 Ruiqi Zhao et al. This is an open access article distributed under the Creative Commons Attribution License, which permits unrestricted use, distribution, and reproduction in any medium, provided the original work is properly cited.

\begin{abstract}
The spoof surface plasmon polaritons (SSPPs) structure can be used as a sensor in $\mathrm{THz}$ region for the biosensing. The accuracy of resonance and amplitude for sensor is very important for biosensing. The momentum matching of SSPPs determines the resonance position and the gap distance determines the amplitude. For the biomolecular sensing, the sample is positioned between the prism base and the SSPPs structure. The momentum matching condition at the current study does not consider the effect of sample refractive index and the resonance position has a significant error. Here the correction is made to the momentum matching condition which considers the effect of the sample refractive index. A comparative study of surface plasmon resonance (SPR) sensing performance based on frequency and angle variations shows that the sensing sensitivity for frequency region is superior to that of angle region; in the meanwhile, as an application of biosensors, we have detected different types of brain lesions in the frequency range. Furthermore, the reflection amplitude is related to gap size between the prism and SSPPs. The relationship of gap size and reflection amplitude is studied. By using the relationship between gap size and reflection amplitude, the amplitudes at different frequencies or incident angles for different refractivities have the same reflection dips compared to the other published results. The simulation is performed and the results proved the theory.
\end{abstract}

\section{Introduction}

In recent years, basic research and development based on surface plasmon polaritons (SPPs) structures and devices have attracted tremendous research enthusiasm, especially in the field of sensing. SPPs are collective oscillations of electron-electromagnetic coupling at a metal/dielectric interface[1]. Their unique properties make them have a wide range of practical applications, including the guidance and manipulation of light at the nanometer scale, biomacromolecules detection, enhanced transmission of light fields through subwavelength aperture, and the absorption and attenuation of electromagnetic (EM) waves [2-6]. The sensors based on surface plasmon resonance (SPR) are one of the most successful applications of SPR at optical frequencies [7]. Many important biological molecules have rotational, translational, and vibrational responses, which uniquely occur in the $\mathrm{THz}$ band, and $\mathrm{THz}$ radiation is nonionizing radiation, which enables terahertz sensing to have broad application prospects in the field of biology sensing [8]. However, the PEC properties of metallic materials in the $\mathrm{THz}$ region make the conventional SPPs unable to be supported at the metal/dielectric interface [9]. Fortunately, Pendry et al. demonstrated theoretically that this problem can be solved by the concept of $\mathrm{THz}$ structural surfaces $[10,11]$, that is, through the modification of the metal surface, to support a terahertz frequency mimicking SPPs, called the spoof SPPs (SSPPs). The plasma frequency of SSPPs can be easily designed through the geometric parameters of the corrugated surfaces, allowing SSPPs to be used in sensing applications from gigahertz $(\mathrm{GHz})$ to midinfrared frequencies. 
We note that SSPPs sensing has been well discussed in the $\mathrm{THz}$ range [12-23]. For example, $\mathrm{Ng}$ et al. studied the relationship between spectral shift and refractive index of SPPs in $1 \mathrm{D}$ periodic grooves by terahertz time-domain spectroscopy (THz-TDS) [12]. Yao and Zhong proposed a high-order mode of spoof SPPs for periodic metal grooves for ultrasensitive terahertz refractive index sensing [14, 15]. Zhang et al. analyzed the angle dependence of the resonance angle on the refractive index change [16]. Li et al.designed the $\mathrm{THz}$ wave switches based on K-R and Otto configurations using the principle of SPR refractive index sensing [20, 21]. Recently, Niknam et al. and others have introduced a new type of double-corrugated ultrasensitive resonance sensor [22].

Generally, most of the SSPPs-based applications in the above literatures are the sensing of the refractive index of filled fluid; however, firstly, the momentum matching condition does not consider the influence of the sample refractive index, which will result in a significant error in the resonance position and reduce the sensitivity of the sensor. Obviously, it is highly undesirable for biomolecular detection. For example, the research work of Bray et al. [24] proved that there are an estimated 18.1 million new cancer cases (17 million excluding non-melanoma skin cancer) and 9.6 million cancer deaths (9.5 million excluding non-melanoma skin cancer). Therefore, the accuracy of the resonance frequency of the SPR biosensor is crucial to improve the sensitivity of the sensor to detect these diseases earlier. Secondly, almost all designs are only used to study the frequency dependence or angle dependence, and there is no comparison between the performances of frequency sensing and angle sensing. Thirdly, since the SSPPs wave decays exponentially in the direction perpendicular to the interface, the coupling gap distance will vary with the filling refractive index, but, in the existing literature $[12,14,15]$, the sensing of different refractive indexes (RI) is carried out according to the same coupling gap. As the RI of the filling medium increases, the optimal coupling regime is broken, resulting in increasing amplitude and wider absorption peak of reflectivity, which limits the possibility of the RI sensing over a wider range.

In this paper, considering the effect of the sample refractive index (RI), we first modified the momentum matching condition, which will help to improve the accuracy and sensitivity of the "refractive index sensing." Then, we verified the correctness of this modification by comparing the resonant positions of the dispersion curve obtained by CST Microwave Studio and the reflection spectrum calculated by COMSOL software. Furthermore, the optimal coupling gap for different refractive indexes is fitted, and the reflectivity is recalculated based on the fitting gap values. Without the shift of the resonance position, the amplitudes at different frequencies or incident angles for different RI have the same reflection dips. Finally, as an application platform for SSPPs biosensing, we carried out sensing detection on brain cancerous cells. The resonance frequency shifts of normal cells and cancer cells infiltrating the gap and groove were calculated by finite element method (FEM).

\section{Theory and Configuration of SPR- Based Sensing}

We used the conventional Otto prism-coupling configuration to excite SSPPs [25], as shown in Figure 1, which consists of a coupling prism and a metal grating with a onedimensional groove structure, where the period is $p$, the groove depth is $h$, the groove width is $a$, and the gap distance between the surface of the metal grating and the prism base is $g$. In order to excite SSPPs on the metal grating, a prism with a high permittivity $\varepsilon_{p}$ is used to increase the wave vector for phase matching. The sample to be measured with the permittivity $\varepsilon_{d}$ is filled in area I and area II. The merits of such a sensing configuration are that the metal groove can be regarded as a microfluidic channel, which significantly reduces the sample consumption and makes the terahertz sensing of biological molecules possible. Furthermore, since the sample is in a relatively closed channel, the disturbance is reduced to a certain extent, so that the reaction occurring in the microfluidic channel can be monitored more accurately in real time.

The collimated $p$-polarized terahertz beam is injected into the prism and refracted at its base at the internal incident angle $\theta_{\text {int }}$. When the momentum matching condition is satisfied, the evanescent wave formed at the base of the prism interacts with the sample in the gap and groove, and the SSPPs wave with exponential decay will be excited on the structure surface of the metal groove. At the frequency for the excitation of SSPPs, the reflectivity will show a sharp dip, known as the SPR phenomenon. Therefore, the sample filled in the microfluidic channel can be decided by measuring the frequency shifts or angle shifts of reflection spectrum due to the variation of sample RI. In order to couple the evanescent wave into SSPPs, $\theta_{\text {int }}$ must be greater than the critical angle of total reflection. The wave vector of incident light in the prism is $k_{P}^{2}=k_{\perp}^{2}+k_{\|}^{2}$, where $k_{\|}$and $k_{\perp}$ represent the parallel and vertical components of the wave vector, respectively. Note that the conventional excitation of SPR requires that the parallel component of two wave vectors of the evanescent wave, $k_{\|}$, and that of the SSPPs, $k_{\mathrm{SSPPs}}$, satisfy the momentum matching condition $[14,15,23,25,26]$ :

$$
k_{\mathrm{SSPPs}}=k_{/ /}=k_{0} n_{p} \sin \left(\theta_{\mathrm{int}}\right) \text {. }
$$

However, formula (1) is only suitable for the case where regions I and II in Figure 1 are air $(n d=1)$; and when the refractive index nd of the filling medium in regions I and II is $n_{d}>1$, formula (1) cannot reflect the effect of the medium on the momentum matching condition. The consequence of using formula (1) will lead to the deviation of the SPR resonance point. Therefore, in order to fully consider the effect of the filling medium in the microfluidic channel on the momentum matching condition, formula (1) is revised as formula (2) in this paper:

$$
k_{\mathrm{SSPPs}}=k_{/ /}=k_{0}\left(\frac{n_{p}}{n_{d}}\right) \sin \left(\theta_{\text {int }}\right),
$$

where $n_{p}=\sqrt{\varepsilon_{p}}, n_{d}=\sqrt{\varepsilon_{d}}$, and $k_{0}=\omega / c$ is the wave vector in the vacuum. In addition, under the limit of $a, P \ll \lambda$, 


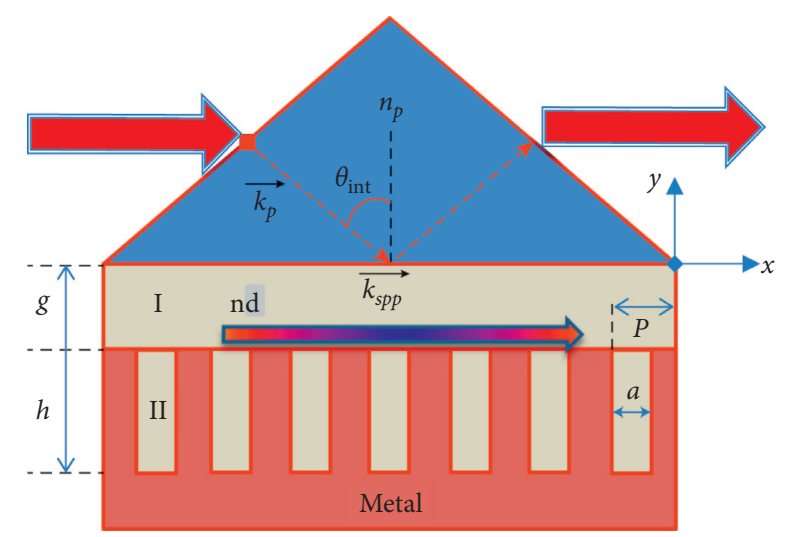

FIGURE 1: The Otto sensing configuration of the SSPPs excitation in the attenuated total reflection regime using a high-permittivity prism and a periodic metal grating. The width, depth, and period of the groove are $a, h$, and $P$, respectively. The gap distance between the prism base and the surface of the metal grating is $g$; $\mathbf{k}_{\mathbf{S P P}}$ is the wave vector of the SSPPs wave. $\theta_{\text {int }}$ is the internal incident angle at the bottom of the prism. The SPR reflectivity is close to zero, and the off-SPR reflectivity is close to one.

according to the effective medium theory and considering the effect of metal loss [11], the dispersion relationship of SSPPs on the surface of the grooved metal can be expressed as follows [27]:

$$
\begin{aligned}
k_{\mathrm{SSPPs}} & =\sqrt{\varepsilon_{d} k_{0}^{2}+\left(\frac{a}{P}\right)^{2} k_{d}^{2} \tan ^{2}\left(k_{d} h\right)}, \\
k_{d} & =k_{0} \sqrt{\varepsilon_{d}}\left(\frac{1+\delta(i+1)}{a}\right)^{(1 / 2)}, \\
\delta & =\left(k_{0} \operatorname{Re} \sqrt{-\varepsilon_{m}}\right)^{-1},
\end{aligned}
$$

where $\varepsilon_{d}$ is the permittivity of the filled medium, $\delta$ is the skin depth of the metal, and $\varepsilon_{m}$ is the complex permittivity of the metal. According to equation (3), the dispersion relation of SSPPs is determined by the subwavelength structure. In other words, the structure of SSPPs has excellent design flexibility, because the effective plasma frequency can be designed within the desired frequency region, which is very desirable for biosensing.

\section{Simulation Results and Discussion}

In order to verify the correctness of formula (2), we use two different numerical simulation pieces of software to compare the results. That is, the dispersion curve of SSPPs wave propagating on the grooved metal at different frequencies is solved by using the eigenmode solver of CST microwave studio software and compared with the reflectivity obtained by the multiphysics field simulation software COMSOL.

Firstly, the dispersion curves of SSPPs supported by 1D periodic rectangular grooves filled with different refractive indexes are analyzed, as shown in Figure 2. The size of periodic unit cell structure is as follows: $a=20 \mathrm{um}$, $h=30 \mathrm{um}$, and $P=60 \mathrm{um}$. The boundary condition is set as follows: the $x$ direction is the periodic boundary, the $y$ direction is set as magnetic wall, and the $z$ direction is set as electrical wall. The fluid refractive indexes corresponding to each dispersion curve of SSPPs in Figure 2 are nd $1=1$, nd $2=1.025$, nd $3=1.05$, nd $4=1.075$, and nd $5=1.1$. The refractive index of the prism is $n_{p}=1.5$. In Figure 2(a), the air line (Line_Air) is the dispersion curve of incident light in the air, while the prism lines (Line_np/nd) are the dispersion curves of parallel wave vectors in the prism when the refractive index of the filling sample is nd. In the low-frequency band, the SSPPs dispersion curve is close to the air line but deviates from the air line when approaching the edge of the first Brillouin region, and the degree of this deviation will increase with the increase of nd. The resonant frequency of SPR sensing is determined by the dispersion curve of incident light in the prism and the dispersion curve of SSPPs supported by the metal surface. That is, when $k_{\text {SSPPs }}=k_{/ /}=k_{0}\left(n_{p} / n_{d}\right) \sin \left(\theta_{\text {int }}\right)$, the prism lines for different refractive indexes will intersect the dispersion curves of SSPPs, and each intersection point represents the SPR frequency of different refractive indexes nd. As shown in Figure 2(b), considering formula (2), the resonance points corresponding to the refractive indexes nd1, nd2, nd3, nd4, and nd5 are points $\mathrm{A}, \mathrm{B}, \mathrm{C}, \mathrm{D}$, and $\mathrm{E}$, respectively. Moreover, the prism line (Line_np/nd) will become closer to the air line (Line_Air) as nd gradually increases. Obviously, compared with the resonance points $\mathrm{A}, \mathrm{B} 1, \mathrm{C} 1, \mathrm{D} 1$, and E1 obtained by formula (1), the frequency shift $\Delta f_{\text {res }}$ caused by the variation $\Delta$ nd of refractive index in formula (2) will be larger. From the perspective of sensing, formula (2) itself implies that its real sensitivity is higher. However, the influence of refractive index nd on momentum matching condition is not considered in the existing literature [13-16, 19, 20, 23, 25, 26]. In view of the dispersion curve, its sensitivity is lower than that in the real case.

Next, in order to verify the above numerical discussion and theoretical analysis and perform comparison with the dispersion curve calculated by CST, the reflectivity of SPR sensor when filledwith different and was calculated by using multiphysical field simulation software COMSOL based on finite element method (FEM). The schematic diagram of the simulation unit cell of the periodic structure is shown in Figure 3(a), where $a=20 \mathrm{um}, h=30 \mathrm{um}, P=60 \mathrm{um}$, and $g=140 \mathrm{um}$. The boundary condition in the propagation direction of SSPPs is set to the Floquet period boundary, and the $k$ vector of the Floquet period is set to $k_{F}=k_{/ /}=k_{0}\left(n_{p} / n_{d}\right) \sin \left(\theta_{\text {int }}\right)$. TM polarization wave is incident to the port on the top of the prism at an internal incident angle $\theta_{\text {int }}=60^{\circ}$, and the propagation constant of the incident port is set to $\beta=k_{\perp}=k_{0}\left(n_{p} / n_{d}\right) \cos \left(\theta_{\text {int }}\right)$. In the simulation, the permittivity of the metal is $\varepsilon_{m}=-1.2 \times 10^{5}-j 7.2 \times 10^{5}$. In order to ensure the convergence and accuracy of the simulation results, the finite element mesh size of each domain is extremely refined. Figure 3(b) shows the reflection spectrum of SPR sensor with different nd as a function of incident frequency, where the range of refractive index is consistent with the previous dispersion curve, from 1 to 1.1 , and the interval is 0.025 . On the one hand, it is clear that there is a minimum value of 


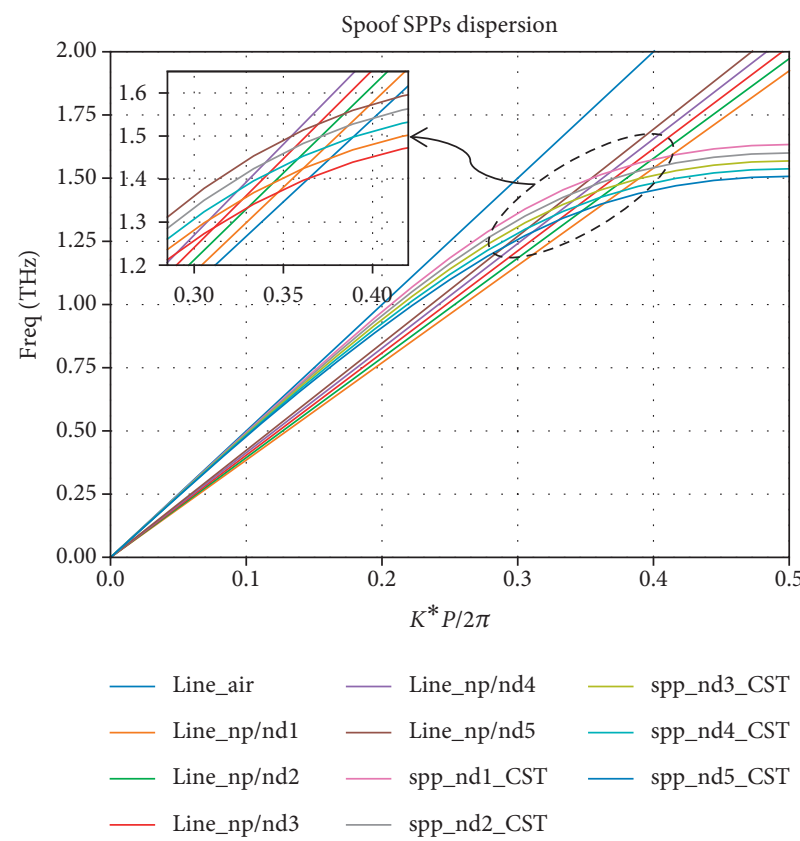

(a)

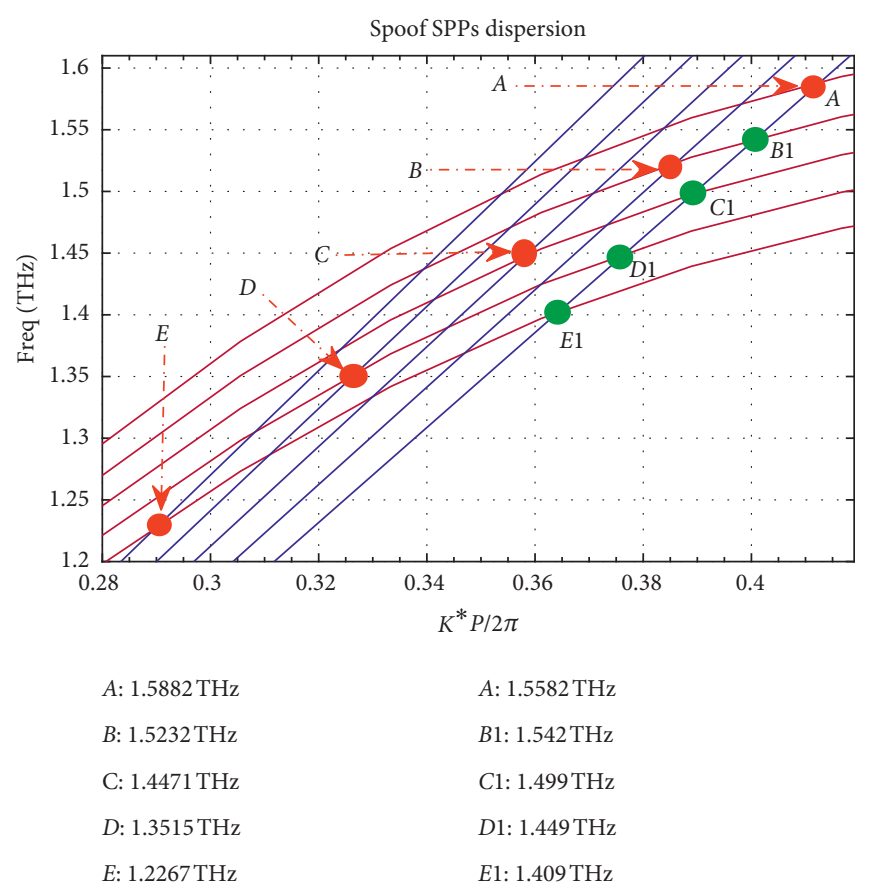

(b)

FiguRE 2: The dispersion curves (a) and the details (b) of the SSPPs supported by periodic metal grooves with $p=60 \mu \mathrm{m}, a=20 \mu \mathrm{m}$, and $h=30 \mu \mathrm{m}$. The filling refractive index is $\mathrm{nd} 1=1, \mathrm{nd} 2=1.025, \mathrm{nd} 3=1.05$, nd $4=1.075$, and $\mathrm{nd} 5=1.1$, respectively. The refractive index of prism is 1.5 .

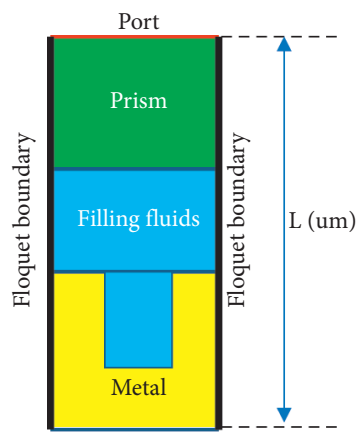

(a)

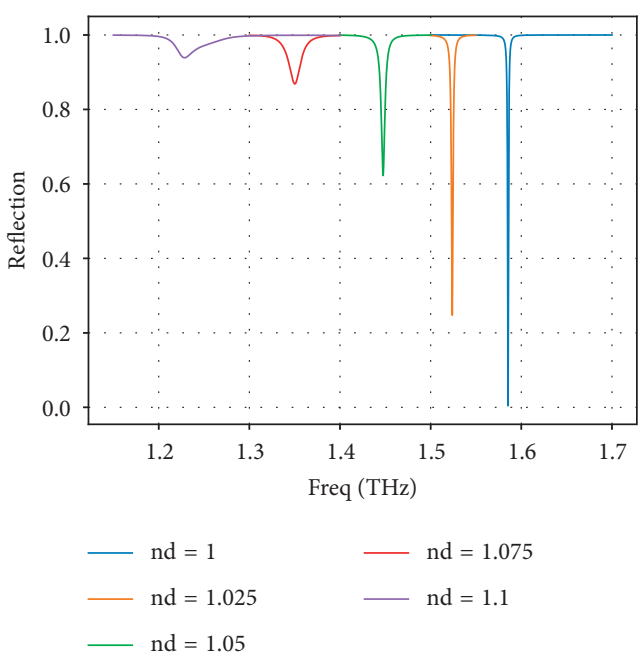

(b)

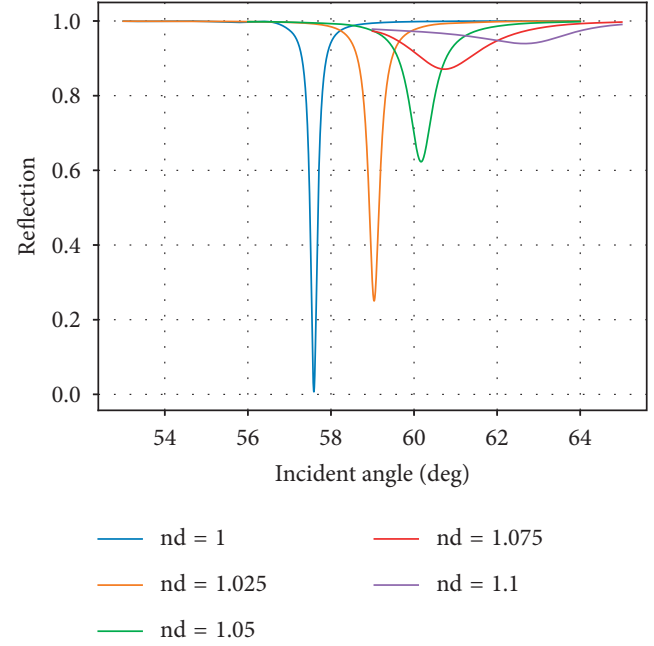

(c)

FIgURE 3: The unit cell structure (a) of 1D periodic groove and the reflectivity spectrums as a function of resonance frequency (b) and resonance angle (c) for the fluids filled with different refractive indexes.

reflectivity in the reflection spectrum of Figure 3(b), which, respectively, corresponds to the resonance frequency of SSPPs excited at different nd. On the other hand, the resonance point of the reflectivity curve for the different nd in Figure 3(b) is very consistent with the resonance points (A, B, C, D, and E) of the dispersion curve in Figure 2(b), and, with the increase of nd, the resonance frequency gradually red-shifts, which also verifies the necessity and correctness of modifying formula (1) to formula (2).

Furthermore, in order to compare the angle dependence and frequency dependence of SPR sensing at the sensitivity level, taking the resonance frequencies in the dispersion curves of Figure 2 as the incident frequencies, we solved the relationship between the reflectance curves and the incident 
angles, as shown in Figure 3(c). It is clear that the reflectivity at each resonance angle $\theta_{\text {res }}$ also has a distinct dip, but the resonance angle is proportional to the refractive index. The detailed values of the resonance frequency $f_{\text {res }}$ and resonance angle $\theta_{\text {res }}$ in Figure 3(b) and 3(c) are shown in Table 1.

From Figures 3(b) and 3(c), it can be seen that as the refractive index of the filled medium deviates from the initial value $(\mathrm{nd}=1)$ corresponding to the optimal coupling gap $(g=140 \mathrm{um})$, the absorption peak of the reflection spectrum begins to broaden and become shallower. This variation of absorption peak can be attributed to the increase of filling refractive index, which not only affects the dispersion curve of SSPPs but also affects the penetration depth of evanescent wave from the prism base into medium. The comparison in optics index between the prism and its contacting sample will be less due to the increase in the refractive index, which will cause a more extension of evanescent wave into the sample gap, and this expansion will lead to an extra reflection on the surface of the metal grating. That is, the approximately optimal sample gap adopted is only applicable to the structure with the specific initial nd. Hence, when the coupling gap departs from its optimal value $g_{\text {opt }}$, the optimal coupling state will be broken. The more the coupling gap deviates from $g_{\text {opt }}$, the more thoroughly the optimal coupling state broken is. The broken coupling leads to a poorer coupling efficiency and further appears on the reflection spectrum as a broader, shallower reflectivity dip.

Therefore, in order to study the influence of the gap on SSPPs, we first analyzed the reflectivity of different coupling gaps at nd $=1.025$, as shown in Figure 4. Figure 4(a) represents reflectivity as a function of frequency variation, while Figure 4(b) represents reflectivity as a function of incident angle variation. It was apparent that the coupling gap only affects the coupling efficiency of SSPPs, which is reflected in the amplitude of the reflectivity, but has no effect on $f_{\text {res }}$ and $\theta_{\text {res }}$. That is, for a certain nd, there is an optimal coupling gap $g_{\text {opt }}$, which corresponds to the reflectance curve with the smallest and narrowest absorption peak. For example, the optimal coupling gap when nd $=1.025$ is $g_{\text {opt }}=162 \mathrm{um}$. The electric field of SSPPs with different nd is different, which is the fundamental reason why the coupling gap affects the coupling efficiency of SSPPs. The insets in Figure 4(a) and 4(b) from left to right are the surface electric field distribution for different coupling gaps $g=130 \mathrm{um}, g=140 \mathrm{um}$, and $g=162 \mathrm{um}$, when the refractive index is 1.025 . According to the inset, the electric field for the optimal coupling gap is the strongest, and the larger the coupling gap deviates from the optimal coupling gap, the weaker the electric field is. For example, the maximum values of the electric field for $g=162 \mathrm{um}, g=140 \mathrm{um}$, and $g=130 \mathrm{um}$ in the insets of Figures $4(\mathrm{a})$ and $4(\mathrm{~b})$ are $4.41 \times 10^{5}(\mathrm{~V} / \mathrm{m})$, $3.82 \times 10^{5}(\mathrm{~V} / \mathrm{m})$, and $3.44 \times 10^{5}(\mathrm{~V} / \mathrm{m})$ and $4.6 \times 10^{5}$ $(\mathrm{V} / \mathrm{m}), \quad 3.98 \times 10^{5}(\mathrm{~V} / \mathrm{m}), \quad$ and $3.43 \times 10^{5}(\mathrm{~V} / \mathrm{m})$, respectively.

According to the above analysis, the sample gap has an optimal value for the highest coupling efficiency with a given refractive index. Here, the optimal sample coupling gaps were obtained by comparing the peak values of the reflectivity at the resonance points with different gap distances adopted (not shown in paper). Through several simulations, the fitting curve of the optimal sample coupling gap for different nd was obtained, as shown in Figure 5. Obviously, with the increase of nd, $g$ increases exponentially. This is because the optimal sample coupling gap is governed by the composite effect of the confinement property of SSPPs and the extension of evanescent wave from the prism base into the sample. Firstly, the complete expression of the evanescent wave is

$$
E_{1}=E_{0} e^{-\alpha_{1} z} F(x)
$$

where $\quad F(x)=e^{j\left(\omega t-\beta_{1} x\right)} \quad$ and $\quad \alpha_{1}=k_{0} n_{p}$ $\sqrt{\left(\sin \left(\theta_{\text {int }}\right)\right)^{2}-\left(n_{d} / n_{p}\right)^{2}}$. Secondly, the transverse electric fields $\left(E_{2}\right)$ of spoof SPPs mode decay exponentially away from the corrugated metal surface and can be expressed as $[28,29]$

$$
E_{2}=\left(\frac{-\alpha_{2}}{j \omega \varepsilon_{0}}\right) A e^{-\alpha_{2} z} e^{-j \beta_{2} x},
$$

where $\alpha_{2}=k_{0} \sqrt{\left(n_{p}\left(\sin \left(\theta_{\text {int }}\right)\right)\right)^{2}-n_{d}^{2}}$. The extension of the evanescent wave is defined as the attenuation length of electromagnetic field to $1 / e$, which is expressed as follows:

$$
L_{1}=\frac{1}{k_{0} \sqrt{\left(n_{p}\left(\sin \left(\theta_{\text {int }}\right)\right)\right)^{2}-n_{d}^{2}}} .
$$

Similarly, the confinement of SSPP waves, defined as attenuation length of electromagnetic field to $1 / e$, is expressed as follows:

$$
L_{2}=\frac{1}{k_{0} \sqrt{\left(k_{\text {SSPS }} / k_{0}\right)^{2}-n_{d}^{2}}},
$$

where $k_{0}=\omega / c$ and $k_{\text {SSPPs }}$ is the wave vector of SSPPs obtained by equation (3). According to the resonance frequencies for the different refractive indexes in Table 1, the curve of $L 1+\mathrm{L} 2$ can be obtained by calculation, as shown in Figure 5, and compared with the optimal coupling gap. On the one hand, the electromagnetic field of SSPPs decays exponentially in the direction perpendicular to the metal surface, and the attenuation will slow down with the increase of nd [30]; on the other hand, for an incident wave of a certain frequency, the evanescent wave at the prism base is a bound state, and the depth of its electromagnetic field extending into the gap is limited, but this limitation will be weakened with the increase of nd. These two aspects will yield a small amount of the "extended" electromagnetic field approaching the edges of the metal groove, which lead to an extra reflection, resulting in a poorer coupling efficiency than the optimal coupling regime. In addition, since the curve trends of $L 1+\mathrm{L} 2$ and $g_{\text {opt }}$ are consistent and $L 1+\mathrm{L} 2$ can be calculated by formulae (8) and (9), in order to show and accurately obtain the existence of an optimum prismmetal gap, we also have fitted the relationship between $L 1+\mathrm{L} 2$ and $g_{\text {opt }}$, which can be expressed as

$$
G(L 1, L 2)=\alpha(L 1+L 2)^{n},
$$


TABle 1: Data comparison based on two numerical methods.

\begin{tabular}{|c|c|c|c|c|c|}
\hline \multirow{2}{*}{ Case } & & \multirow{2}{*}{$\mathrm{CST}-\mathbf{f}_{\text {res }}(\mathrm{THz})$} & \multicolumn{3}{|c|}{ COMSOL } \\
\hline & & & $f_{\text {res }}(\mathrm{THz})$ & $g_{\text {opt }}(\mathrm{um})$ & $\theta_{\text {res }}(\mathrm{deg})$ \\
\hline \multirow[b]{5}{*}{ 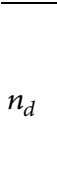 } & 1 & 1.5882 & 1.5854 & 140 & $57.46^{\circ}$ \\
\hline & 1.025 & 1.5232 & 1.5238 & 162 & $59.05^{\circ}$ \\
\hline & 1.05 & 1.4471 & 1.4475 & 190 & $60.12^{\circ}$ \\
\hline & 1.075 & 1.3515 & 1.3501 & 247 & $60.88^{\circ}$ \\
\hline \multirow{2}{*}{\multicolumn{2}{|c|}{1.1}} & 1.2267 & 1.2285 & 325 & $61.68^{\circ}$ \\
\hline & & $f_{\text {res }}=-3.57 n_{d}+5.18$ & \multicolumn{2}{|c|}{$f_{\text {res }}=-3.55 n_{d}+5.15$} & $\theta_{\text {res }}=0.74 n_{d}+0,270.74$ \\
\hline \multicolumn{2}{|c|}{ Sensitivity } & $3.57 \mathrm{THz} / \mathrm{RIU}$ & \multicolumn{2}{|c|}{$3.55 \mathrm{THz} / \mathrm{RIU}$} & $0.74 \mathrm{rad} / \mathrm{RIU}$ \\
\hline
\end{tabular}

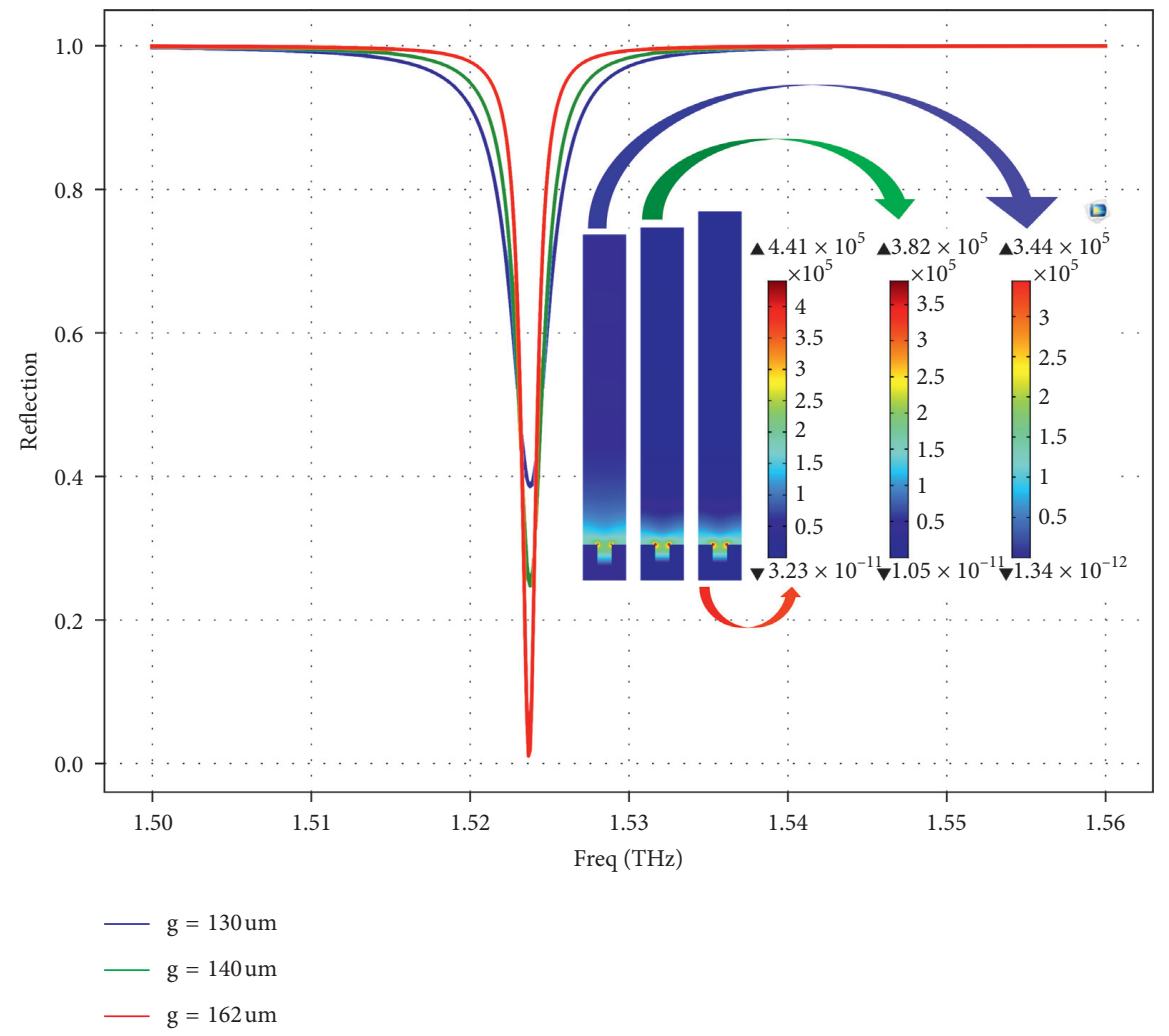

(a)

Figure 4: Continued. 


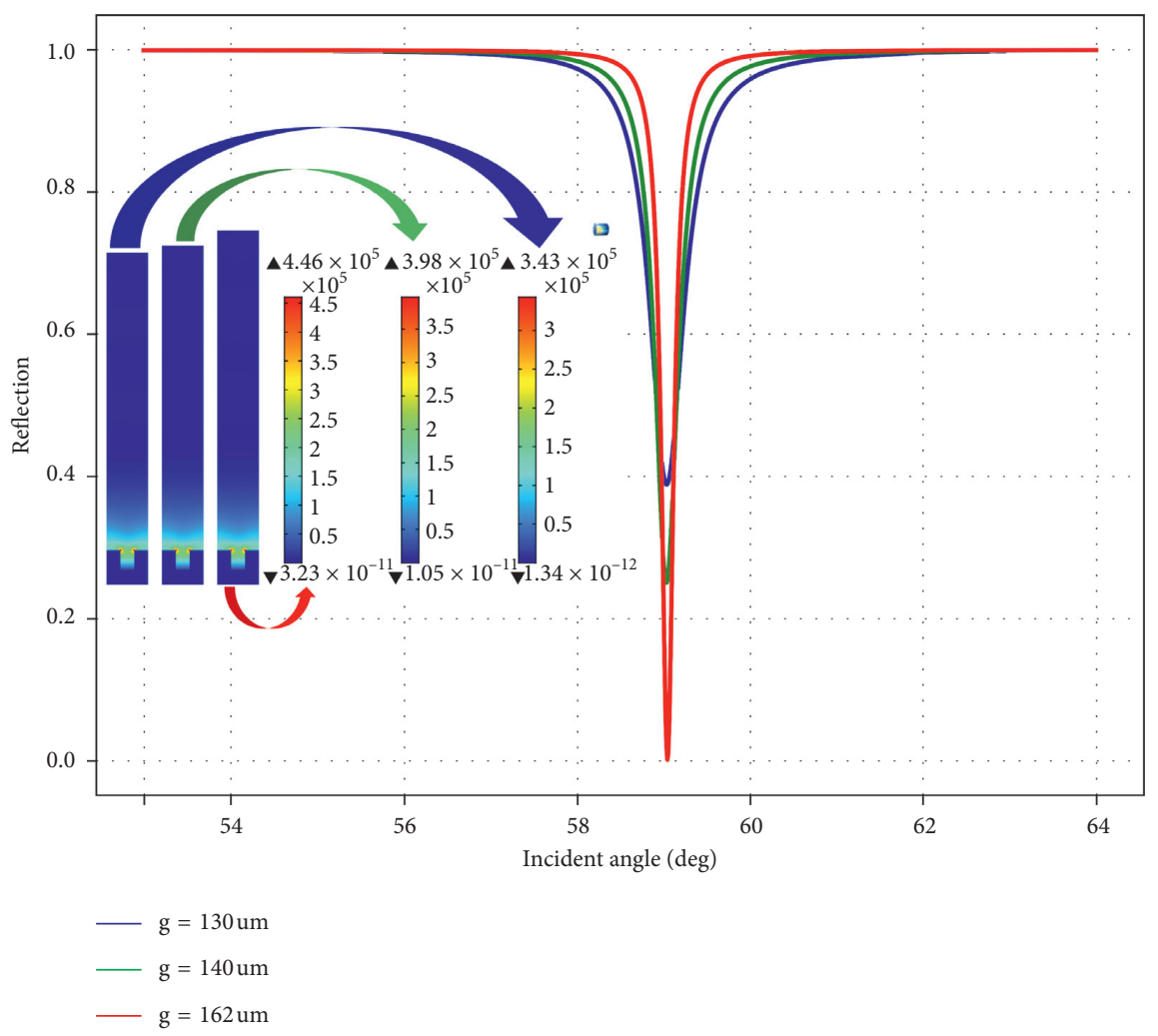

(b)

FIGURE 4: Reflectivity spectrums as a function of resonance frequency (a) and incident angle (b) and the electric field amplitude distribution of different gap distances at $\mathrm{nd}=1.025$.

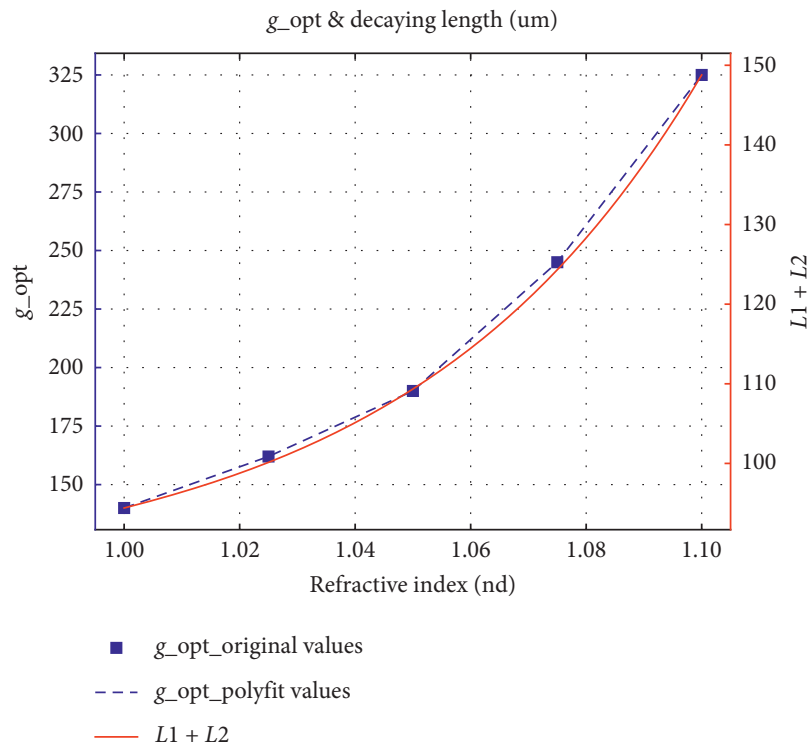

Figure 5: The fitting curve of the optimal gap and the attenuation curve of the electromagnetic field under different filled refractive indexes.

where $G(L 1, L 2)$ is the optimal coupling gap, which is a function of L1 and L2, and alpha and $n$ are the fitting coefficients of the structure. $\alpha=0.042$ and $n=1.788$. According to the data in Figure 5, under the condition that each nd is matched with its optimal coupling gap, we simulated the reflection spectra of different refractive indexes as a function of resonance frequency and resonance angle, and the results are illustrated in Figures 6(a) and 6(b), respectively. Obviously, since gap only affects the amplitude of the reflectivity and has no effect on the resonance point ( $f_{\text {res }}$ and $\theta_{\text {res }}$ ), when the coupling gaps of SSPPs with different nd are optimal, the resonance frequencies of SPR in 


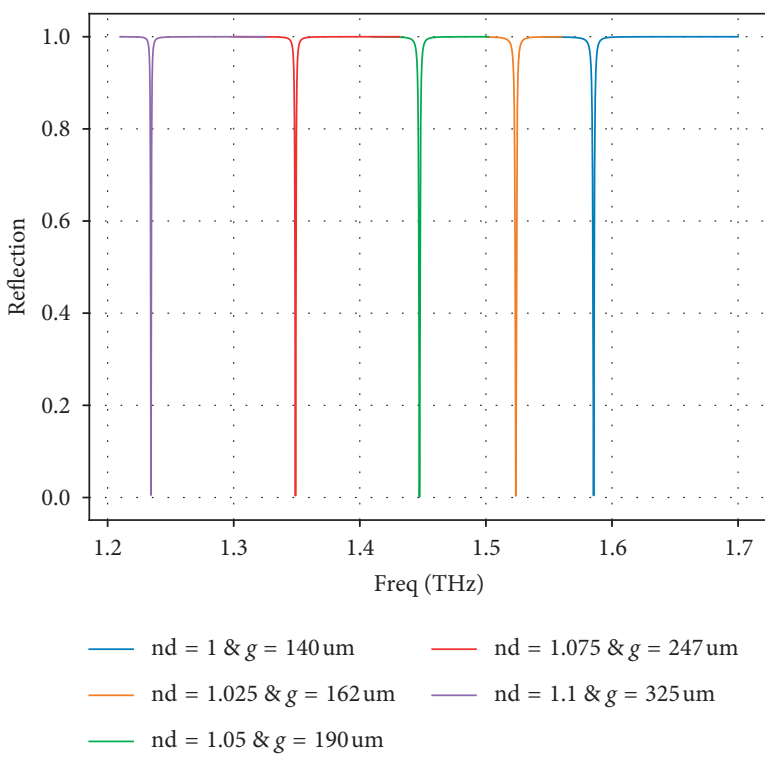

(a)

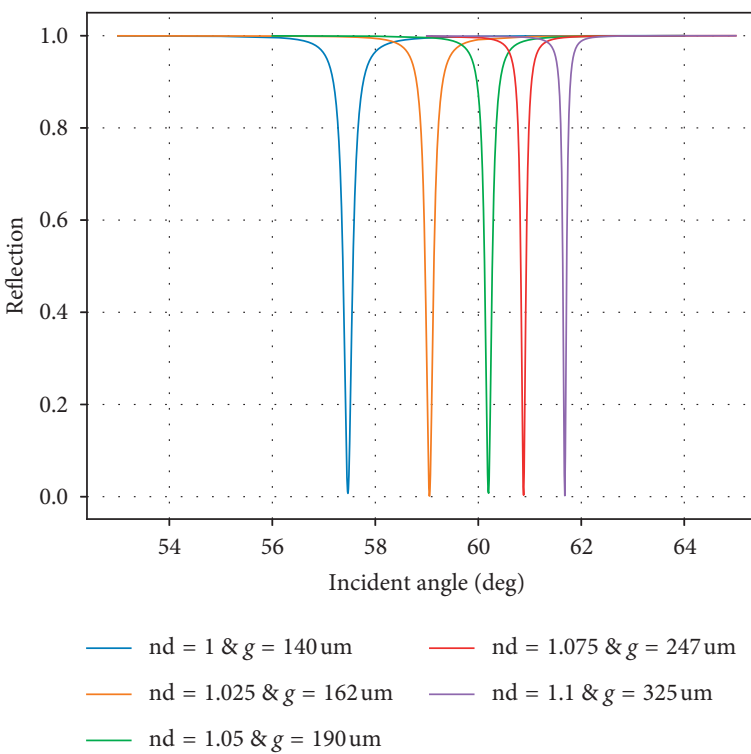

(b)

Figure 6: The reflectivity spectrum as a function of resonance frequency (a) and resonance angle (b) for the fluids filled with different refractive indexes under the optimal gaps.

Figures 3(b) and 6(a) are the same and the resonance angles in Figures 3(c) and 6(b) are also consistent, but the minimum values of the absorption peak of reflectivity in Figure 6 are all less than $0.05 \%$, almost close to 0 , and the absorption peak is sharper, and the width of the absorption peak is narrower, representing a higher Q-factor and higher sensitivity.

Here, the mutual verification of resonant frequencies obtained by CST and COMSOL and the comparison of frequency dependence and angle dependence of SPR sensing are carried out, respectively, as shown in Table 1. It is clear that the SPR resonance frequencies obtained by the two numerical methods are basically the same, which verifies the correctness of the correction of formula (2) in our previous theoretical analysis. Furthermore, according to the previous theoretical analysis, compared to formula (1), the revised formula (2) itself implies the fact that the sensitivity is higher and up to $3.57 \mathrm{THz} / \mathrm{RIU}$ (refractive index unit), which is much more sensitive than in previous work. For example, the sensitivities in literature $[14,15,23]$ are $2.27 \mathrm{THz} / \mathrm{RIU}$, $2.57 \mathrm{THz} / \mathrm{RIU}$, and $0.4 \mathrm{THz} / \mathrm{RIU}$, respectively.

In order to more intuitively reflect the comparison of the two numerical results in Table 1, we draw the fitting curves of the relationship between the resonance frequency and resonance angle and the refractive index of the filling fluid. As shown in Figure 7, the two fitting curves of the resonance frequency are very close, and, according to the fitting curve, we can easily obtain the resonance frequencies for different nd, and, combining formulae (6) and (7), we can calculate the curve of $L 1+\mathrm{L} 2$, so as to obtain the approximate optimal coupling gaps based on the proportional relationship of the vertical axis, which provides a new method for determining the optimal sample coupling gap. Moreover, the absolute value $0.74 \mathrm{rad} / \mathrm{RIU}$ of the slope of the fitting curve of resonance angle is much less than the slope value $3.57 \mathrm{THz} / \mathrm{RIU}$

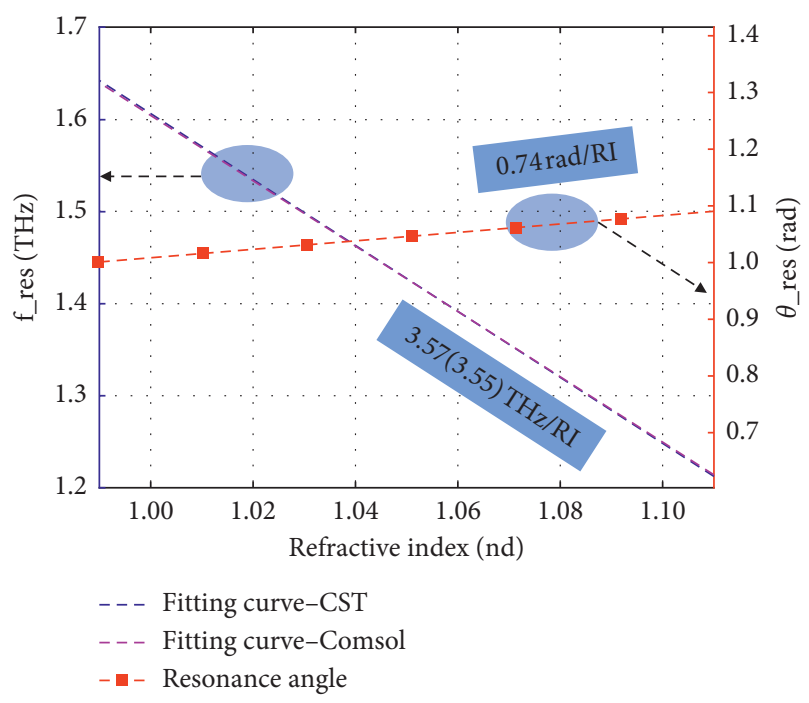

FIGURE 7: Fitting curve of resonance frequency and resonance angle and refractive index.

of resonance frequency, which indicates that the sensing performance of SPR based on frequency variation is better than that of angle variation.

Unfortunately, millions of people die from cancer every year. Therefore, the detection of highly sensitive cancerous cells has important biological significance $[8,24]$. Here, as an application of our proposed biosensing platform based on the improved formula, this article ends with the detection of brain lesions. That is, the resonance frequency shifts of normal cells and cancer cells infiltrating the gap and groove were calculated by finite element method. Here the refractive index (RI) of the brain cancer cells is shown in Table 2 $[31,32]$. For a solution with $100 \%$ distilled water, the refractive index (RI) is 1.333, which acts as an external 
TABLE 2: The refractive index of various brain tissues.

\begin{tabular}{lc}
\hline Brain lesions tissues & Refractive index \\
\hline CSF (reference) & 1.3333 \\
Wall of solid sclerosis & 1.3412 \\
Multisclerosis & 1.3425 \\
Oligodendroglioma & 1.3531 \\
Gray matter & 1.3951 \\
White matter & 1.4121 \\
Low-grade glioma & 1.4320 \\
Medulloblastoma & 1.4412 \\
Glioblastoma & 1.4470 \\
Lymphoma & 1.4591 \\
Metastasis & 1.4833 \\
\hline
\end{tabular}

reference, and here the CSF with RI of 1.3333 is assumed as an internal reference.

According to formula (3), the plasma frequency can be designed by redesigning the geometric parameters of the corrugated metal, so as to effectively excite SSPPs on the surface of periodic structure when the cancer cells and normal cells are infiltrated into the gaps and grooves. It can be seen from Figure 2(a) that the dispersion curve of SSPPs will deviate further apart from the light line (Line_Air) as the refractive index increases, but the parallel component $k_{/ /}$of the wave vector in the prism is closer to the light line. Note that when nd increases to a certain value (of course, since SSPPs are a slow wave, i.e., $k_{\mathrm{SSPP}} / k_{0}>n_{d}$, the theoretically detectable sample maximum refractive index is $\left.n_{d}<\sqrt{n_{p} \sin \left(\theta_{\text {int }}\right)}\right)$, there will be no intersections between the parallel component of the wave vector in the prism and the dispersion curve of SSPPs. That is, SSPPs cannot be effectively excited. Here, when the filling sample is the brain cancer cells, in order to effectively excite SSPPs, we redesigned the geometric dimensions of the metal grooves as follows: $a=20 \mathrm{um}, h=50 \mathrm{um}$, and $P=60 \mathrm{um}$. The permittivity of the prism is $\varepsilon_{p}=n_{p}^{2}=11.63$.

As mentioned in the previous theory and simulation analysis, the sensing sensitivity of SPR in the frequency domain is better than that in the angle domain, so next we will mainly conduct the reflection spectra of brain lesions in the frequency region. Here, we carry out simulation analysis for the two cases, $\theta_{\text {int }}=45^{\circ}$ and $\theta_{\text {int }}=60^{\circ}$, and when the metal groove and the gap space under the prism were infiltrated with brain cancer cells, the reflectivity at different incident frequencies is calculated by COMSOL, respectively, as illustrated in Figures 8(a) and 8(b); the reflectance spectrum of the proposed sensing device manifests that the SPR frequency of cancer cells will red-shift as the refractive index increases. Note that the reflection spectrums in Figure 8 are calculated under the optimal coupling gaps corresponding to different cells, and the determination of $g_{\mathrm{opt}}$ is similar to the process discussed above. In addition, in order to more clearly show the change of resonance frequency in Figure 8, we draw the resonance positions of brain lesions at different incident angles in Figure 9. It can be seen from Figure 9 that the resonant frequency corresponding to 45 degrees is lower than that corresponding to 60 degrees. This is because the larger the incident angle, the larger the wave vector $k$ of the SSPPs, and the smaller the frequency of the SPR.

The sensitivity is a key factor in evaluating the efficiency of a sensor. For the sensitivity of the refractive index sensor, we define it as the frequency shift value divided by the refractive index change of the biological sample:

$$
S=\frac{\Delta f_{\text {res }}}{\Delta n_{d}},
$$

where $\Delta f_{\text {res }}=f_{\text {res }}$ (cancerous cell) $-f_{\text {res }}$ (normal cell) and $\Delta n_{d}=n_{d}$ (cancerous cell $)-n_{d}$ (normal cell). Besides, the figure of merits (FOM) is another significant indicator of sensor performance, which can be defined as

$$
\mathrm{FOM}=\frac{S}{\Delta f_{1 / 2}},
$$

where $\Delta f_{1 / 2}$ is the spectral full width at half maximum of the reflection dip. Table 3 shows the important performance parameters of designed SSPPs sensors for various cancers at different incident angles (45deg and 60deg). The maximum values of the sensitivity and FOM when $\theta_{\text {int }}=45^{\circ}$ are up to $2 \mathrm{THz} / \mathrm{RIU}$ and 3337, respectively, while the maximum values of the sensitivity when $\theta_{\text {int }}=60^{\circ}$ are $0.9347 \mathrm{THz} / \mathrm{RIU}$ and corresponding FOM of about 360 can be also obtained. Obviously, the sensitivity when the incident angle is 45 degrees is better than that when the incident angle is 60 degrees. The electric field confinement ability of the SSPPs on the surface of the periodic groove structure at two incident angles is the main reason for this difference in sensing performance. Here, the CSF are considered as a reference and we calculated the electric field intensity $\operatorname{normE}=\sqrt{\mathrm{abs}(E x)^{2}+\mathrm{abs}(E y)^{2}+\mathrm{abs}(E z)^{2}}$, as shown in Figure 10. It is clear that the electric field for 45 degrees is stronger than that for 60 degrees; that is, the interaction between the biological sample and SSPPs at 45 degrees is stronger than that at 60 degrees, which leads the SSPPs to be more sensitive to changes in the dielectric environment near the groove surface.

In addition, in order to more clearly describe the detection effect of different degrees of brain lesions, we plotted the relationship between $S$ and FOM with brain cancer cells in Figures 11(a) and 11(b). It can be seen intuitively from Figure 11 that this structure has excellent performance in detecting different degrees of brain lesions, and, with the deterioration of cancerization, the detected $S$ value and FOM value will be larger.

Finally, Table 4 compares the sensitivity and FOM value of proposed structure in this research with the previous designed sensors operated in the $\mathrm{THz}$ region. The results show that the performance of the biosensor based on the modified momentum matching formula proposed in this paper is far superior to previous work. Owing to its high sensing performance above, it could be believed that the proposed SSPPs sensor based improved momentum matching condition would provide effective guides for achieving high-sensitivity RI biosensor in $\mathrm{THz}$ region. 


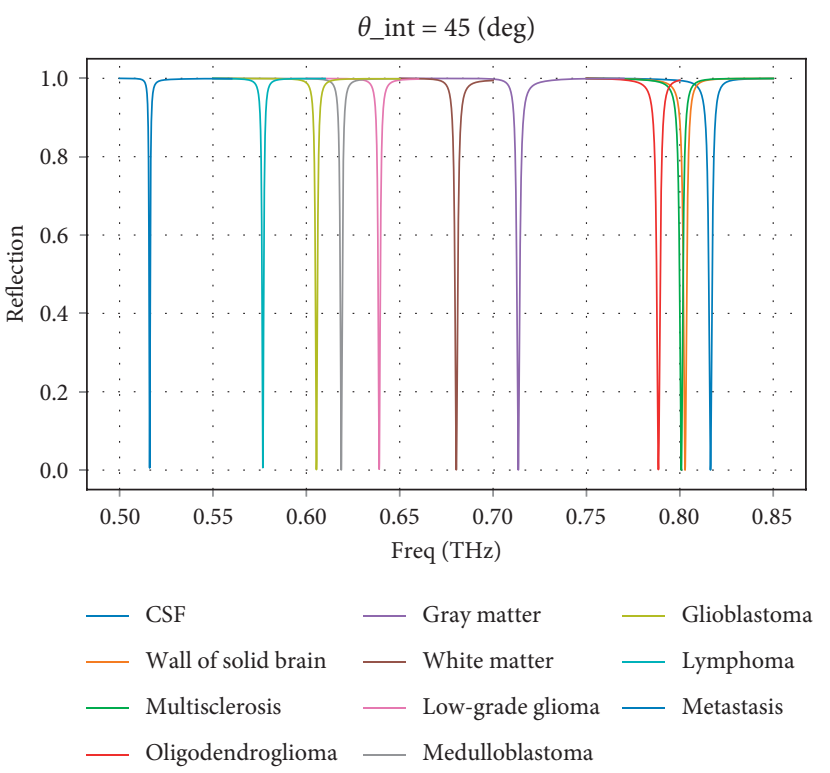

(a)

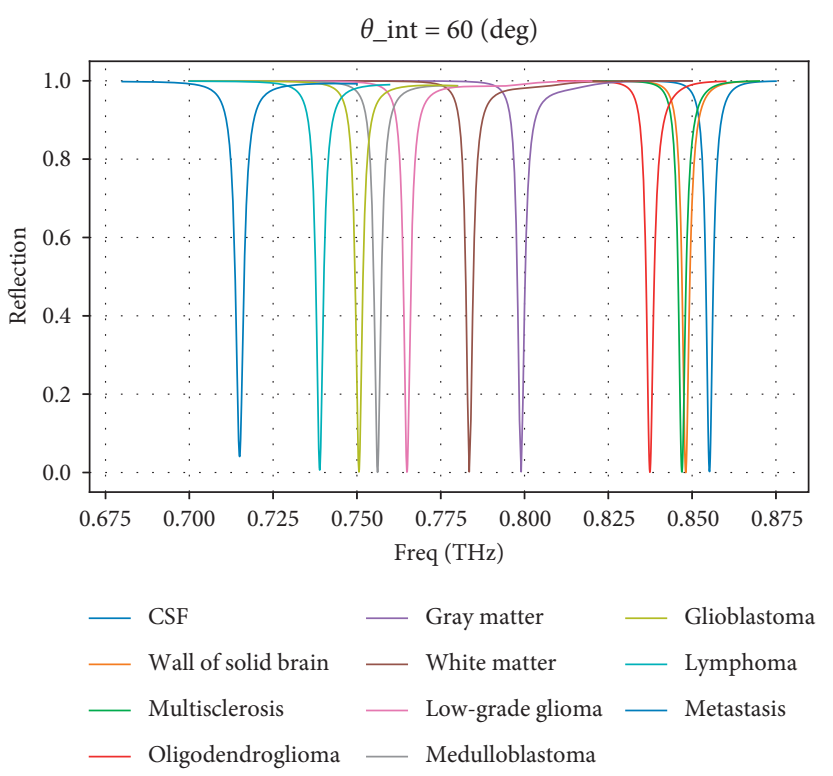

(b)

Figure 8: The reflection spectrums when the sample layer is filled with different types of brain cancerous cells at $\theta_{\text {int }}=45^{\circ}$ (a) and $\theta_{\text {int }}=60^{\circ}$ (b).

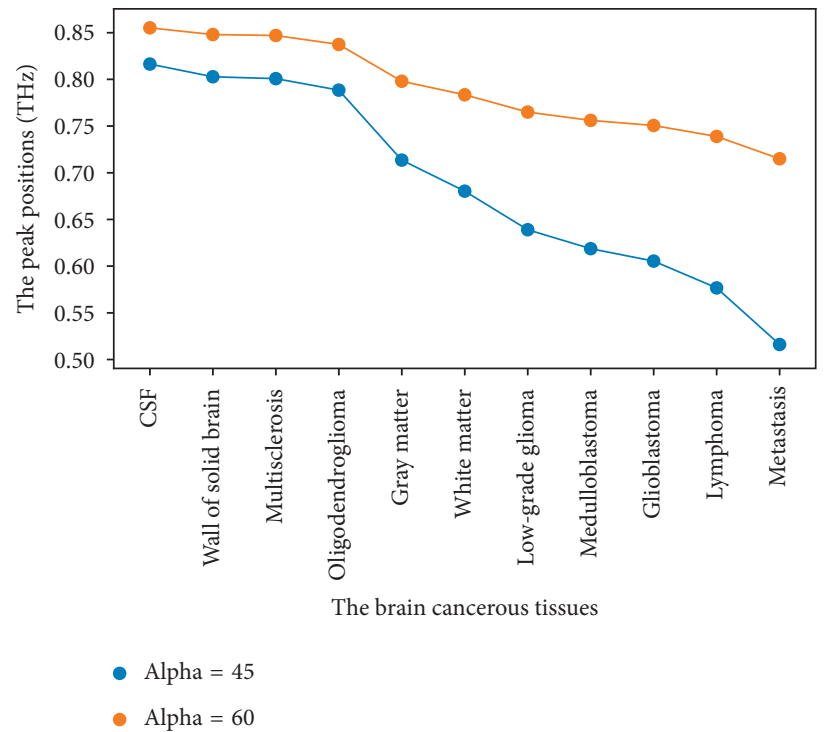

FIgURE 9: The resonant peak positions for brain lesions at different incident angles.

TABLE 3: The performance parameters S and FOM of designed SSPPs sensors for various cancers at different incident angles.

\begin{tabular}{|c|c|c|c|c|c|c|c|c|c|c|}
\hline \multirow{2}{*}{$\begin{array}{l}\text { Brain tissues } \\
\text { samples }\end{array}$} & \multicolumn{5}{|c|}{$\theta_{\text {int }}=45^{\circ}$} & \multicolumn{5}{|c|}{$\theta_{\text {int }}=60^{\circ}$} \\
\hline & $f_{\text {res }}(\mathrm{THz})$ & $\Delta f_{1 / 2}(\mathrm{THz})$ & $\Delta f_{\text {res }}(\mathrm{THz})$ & $\begin{array}{l}\text { S (THz/ } \\
\text { RIU) }\end{array}$ & $\begin{array}{c}\text { FOM } \\
(1 / \text { RIU })\end{array}$ & $f_{\text {res }}(\mathrm{THz})$ & $\Delta f_{1 / 2}(\mathrm{THz})$ & $\Delta f_{\text {res }}(\mathrm{THz})$ & $\begin{array}{l}\text { S (THz/ } \\
\text { RIU) }\end{array}$ & $\begin{array}{c}\text { FOM } \\
(1 / \text { RIU })\end{array}$ \\
\hline CSF (reference) & 0.8164 & 0.0018 & 0 & 0 & 0 & 0.8552 & 0.0023 & 0 & 0 & 0 \\
\hline $\begin{array}{l}\text { Wall of solid } \\
\text { sclerosis }\end{array}$ & 0.8028 & 0.0019 & 0.0136 & 1.7215 & 906 & 0.848 & 0.0024 & 0.0072 & 0.9114 & 380 \\
\hline Multisclerosis & 0.8008 & 0.0018 & 0.0156 & 1.6957 & 942 & 0.847 & 0.0024 & 0.0082 & 0.8913 & 371 \\
\hline Oligodendroglioma & 0.7885 & 0.0019 & 0.0279 & 1.4091 & 742 & 0.8374 & 0.0025 & 0.0178 & 0.899 & 360 \\
\hline Gray matter & 0.7136 & 0.0017 & 0.1028 & 1.6634 & 978 & 0.798 & 0.0024 & 0.0572 & 0.9256 & 386 \\
\hline White matter & 0.6803 & 0.0016 & 0.1361 & 1.7272 & 1080 & 0.7835 & 0.0023 & 0.0717 & 0.9099 & 396 \\
\hline Low-grade glioma & 0.6390 & 0.0012 & 0.1774 & 1.7974 & 1498 & 0.765 & 0.0023 & 0.0902 & 0.9139 & 397 \\
\hline Medulloblastoma & 0.6187 & 0.0012 & 0.1977 & 1.8323 & 1527 & 0.7561 & 0.0023 & 0.0991 & 0.9184 & 399 \\
\hline Glioblastoma & 0.6054 & 0.0012 & 0.2110 & 1.8558 & 1547 & 0.7506 & 0.0026 & 0.1046 & 0.92 & 354 \\
\hline Lymphoma & 0.5767 & 0.0009 & 0.2397 & 1.9054 & 2117 & 0.7389 & 0.0024 & 0.1163 & 0.9245 & 385 \\
\hline Metastasis & 0.5161 & 0.0006 & 0.3003 & 2.0020 & 3337 & 0.7150 & 0.0026 & 0.1402 & 0.9347 & 360 \\
\hline
\end{tabular}




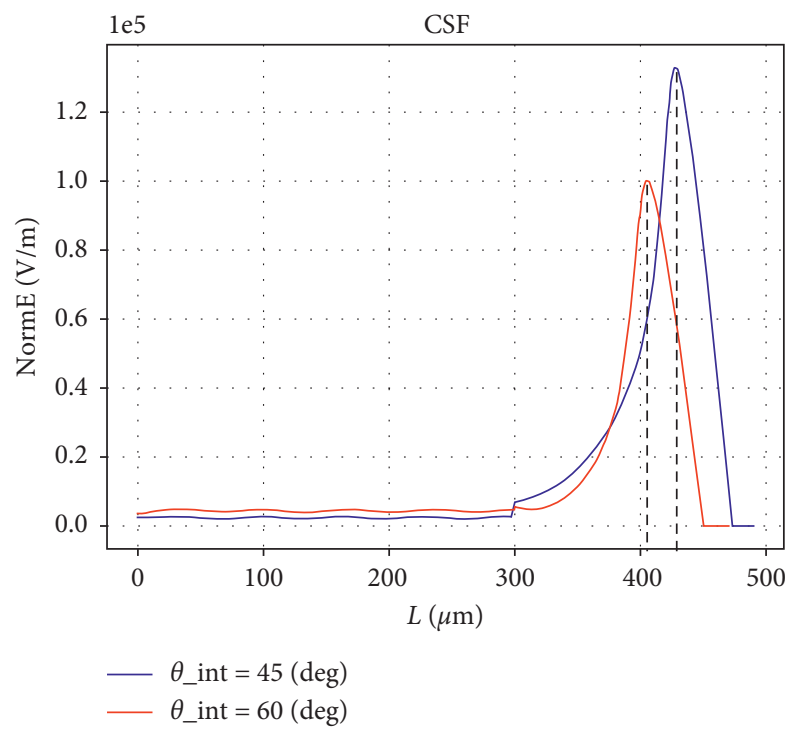

FIGURE 10: Electric field normE perpendicular to the surface of the periodic structure at different incident angles when the biological sample is CSF; $L$ is the total thickness of the prism layer, sample layer, and metal layer.

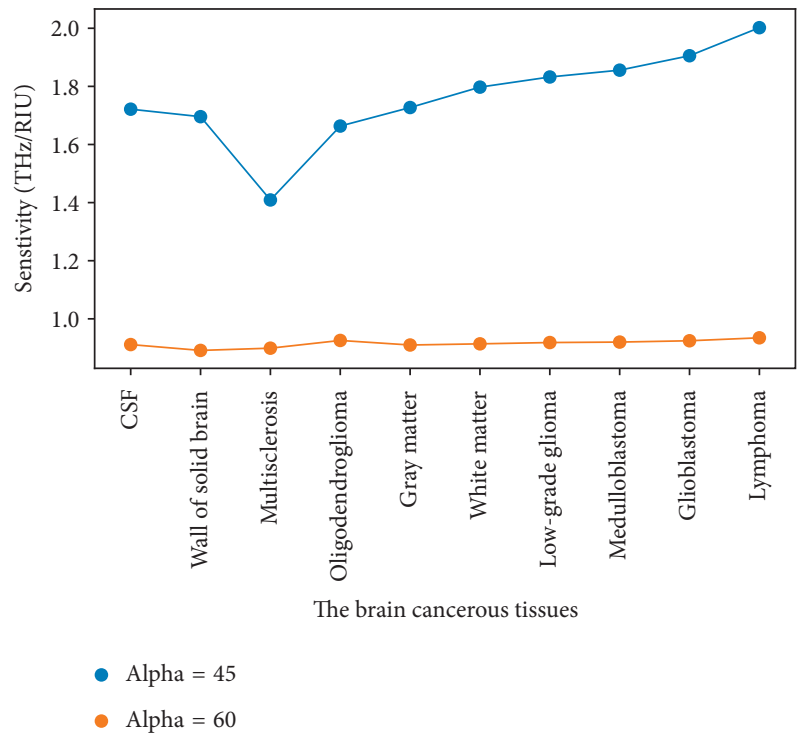

(a)

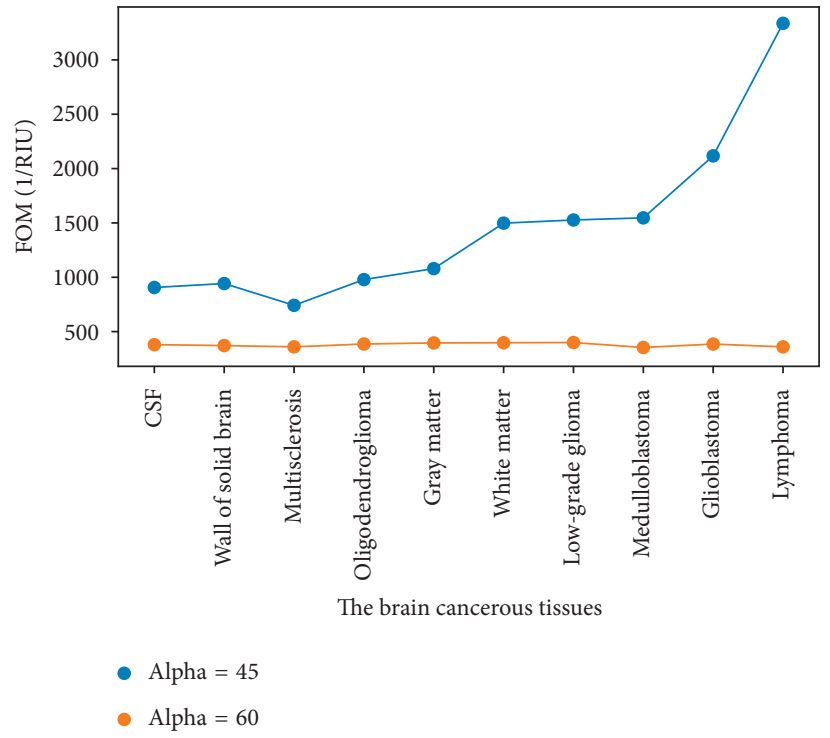

(b)

FIGURE 11: The sensitivity (a) and FOM (b) for different brain cancerous cells at different incident angles.

TABLE 4: The sensitivity comparison of the proposed structure and the latest relevant systems.

\begin{tabular}{lcc}
\hline Reference & S (THz/RIU) & FOM (1/RIU) \\
\hline [33] 2015 & 0.3930 & 19.86 \\
[34] 2017 & 0.3050 & 692 \\
[19] 2017 & 1.9660 & 244 \\
[35] 2018 & 0.4380 & 60.76 \\
[36] 2018 & 1.6870 & 3966,3337 \\
[37] 2019 & 0.5216 & \\
[38] 2020 & 0.9600 & $3.57,2$ \\
This research & & \\
\hline
\end{tabular}




\section{Conclusions}

In summary, we have numerically investigated the performance of $\mathrm{THz}$ SSPPs-based sensing of refractive index changes under the premise that the filling sample refractive index will affect momentum matching condition. Firstly, we modify the dispersion formula of the parallel component of the wave vector in the prism and verify the correctness of the formula from the two aspects of dispersion curve and reflectivity. The results show that reflection amplitude depends on the gap distance. For a given filled sample with a certain nd, there is an optimal coupling gap for minimal reflection amplitude. We fitted the relationship curve between the optimal coupling gap and the filling nd. Then, the reflectivity is calculated on the basis of adjusting the optimal coupling gap distance, so that the coupling of SSPPs with different nd is in the optimal regime; that is, the absorption peak of the reflectivity for each nd is very sharp, and the minimum value of reflectivity is below $0.05 \%$, which realizes the high-sensitivity detection of specific analytes. The SPRbased sensing performance of the frequency region is superior to that of the angle region, and the sensing results of filling fluids with different nd show that the sensitivity of the improved formula (2) is as high as $3.57 \mathrm{THz} / \mathrm{RIU}$ and the corresponding FOM is 3966.

In addition, as a sensing application platform, we conducted the brain lesions sensing with different types of cancerous cells. The numerical results show that the maximum sensitivity is about $2 \mathrm{THz} / \mathrm{RIU}$, and the corresponding FOM is 3337 , which is significantly better than the previously designed sensors that work in the terahertz region.

Moreover, since the response of the SSPPs can be engineered by designing its geometrical sizes, the sensing for biological samples can be performed in the desired frequency range, which is crucial for biological and security sensing based on the terahertz microchannel.

\section{Data Availability}

The data used to support the findings of this study are included within the article.

\section{Conflicts of Interest}

The authors declare no conflicts of Interest.

\section{Acknowledgments}

Thanks are due to Professor Guizhen Lu and other collaborators for their theoretical guidance on this research work. This research was funded by the Beijing Institute of Environmental Laboratory (Grant no. 621802Y030204).

\section{References}

[1] X. Zhang, Q. Xu, and L. Xia, "Terahertz surface plasmonic waves: a review," Advanced Photonics, vol. 2, no. 1, 2020.

[2] E. Pickwell and V. P. Wallace, "Biomedical applications of terahertz technology," Journal of Physics D: Applied Physics, vol. 1, no. 17, pp. 73-81, 2002.
[3] S. Lepeshov, A. Gorodetsky, A. Krasnok, E. U. Rafailov, and P. Belov, "Enhancement of terahertz photoconductive antennas and photomixers operation by optical nanoantennas," Laser \& Photonics Review, vol. 11, 2017.

[4] Q. Yun-Ping, N. Xiang-Hong, B. Yu-Long, and W. XiangXian, "All-optical diode of subwavelength single slit with multi-pair groove structure based on SPPs-CDEW hybrid mode," Acta Physica Sinica, vol. 11, pp. 270-281, 2017.

[5] M. Ç. Ayan, S. Kiriş, A. Yapici et al., "Investigation of cotton fabric composites as a natural radar-absorbing material," Aircraft Engineering and Aerospace Technology, vol. 92, 2020.

[6] M. Ozturk, T. Depci, M. Karaaslan, U. K. Sevim, O. Akgol, and S. Ozdemir Hacioglu, "Synergetic effect of waste tire rubber and mil scale on electromagnetic wave attenuation properties of new generation mortar," Journal of Building Engineering, vol. 30, p. 101249, 2020.

[7] Z. Gao, L. Wu, F. Gao, Y. Luo, and B. Zhang, "Spoof plasmonics: from metamaterial concept to topological description," Advanced Materials, vol. 30, no. 31, p. 1706683, 2018.

[8] D. Cheng, X. He, X. Huang et al., "Terahertz biosensing metamaterial absorber for virus detection based on spoof surface plasmon polaritons," International Journal of RF and Microwave Computer-Aided Engineering, vol. 28, no. 7, p. e21448, 2018.

[9] J. G. Rivas, “The art of confinement," Nature Photonics, vol. 2, no. 3, pp. 137-138, 2008.

[10] J. B. Pendry, "Mimicking surface plasmons with structured surfaces," Science, vol. 305, no. 5685, pp. 847-848, 2004.

[11] F. J. Garcia-Vidal, L. Martín-Moreno, and J. B. Pendry, "Surfaces with holes in them: new plasmonic metamaterials," Journal of Optics A: Pure and Applied Optics, vol. 7, no. 2, pp. S97-S101, 2005.

[12] B. Ng, J. Wu, S. M. Hanham et al., "Spoof plasmon surfaces: a novel platform for THz sensing," Advanced Optical Materials, vol. 1, no. 8, pp. 543-548, 2013.

[13] S. M. Breese and S. A. MAIER, "Terahertz plasmonic surfaces for sensing," Active Plasmonics and Tuneable Plasmonic Metamaterials, pp. 243-260, 2013.

[14] H. Yao and S. Zhong, "High-mode spoof SPP of periodic metal grooves for ultra-sensitive terahertz sensing," Optics Express, vol. 22, no. 21, p. 25149, 2014.

[15] H. Yao, S. Zhong, and W. Tu, "Performance analysis of higher mode spoof surface plasmon polariton for terahertz sensing," Journal of Applied Physics, vol. 117, no. 13, p. 133104, 2015.

[16] Y. Zhang, Z. Hong, and Z. Han, "Spoof plasmon resonance with $1 \mathrm{D}$ periodic grooves for terahertz refractive index sensing," Optics Communications, vol. 340, pp. 102-106, 2015.

[17] Z. Xu and P. Mazumder, "Bio-sensing by mach-zehnder interferometer comprising doubly-corrugated spoofed surface plasmon polariton (DC-SSPP) waveguide," IEEE Transactions on Terahertz Science and Technology, vol. 2, no. 4, pp. 460-466, 2012.

[18] Y.-Q. Liu, L.-B. Kong, C.-H. Du, and P.-K. Liu, "Spoof surface plasmon modes on doubly corrugated metal surfaces at terahertz frequencies," Journal of Physics D: Applied Physics, vol. 49 , no. 23 , p. 235501, 2016.

[19] X. Chen and W. Fan, "Ultrasensitive terahertz metamaterial sensor based on spoof surface plasmon," Scientific Reports, vol. 7, no. 1, 2017.

[20] L. Jiu-sheng, "Switching terahertz wave with grating-coupled Kretschmann configuration," Optics Express, vol. 25, no. 16, p. 19422, 2017.

[21] S.-H. Li and J. Li, "Terahertz switch based on otto configuration using spoof surface plasmon polariton," in Proceedings 
of the 2018 12th International Symposium on Antennas, Propagation and EM Theory (ISAPE), Hangzhou, China, December 2018.

[22] S. Niknam, M. Yazdi, and S. Behboudi Amlashi, "Enhanced ultra-sensitive metamaterial resonance sensor based on double corrugated metal stripe for terahertz sensing," Scientific Reports, vol. 9, no. 1, 2019.

[23] L. Chen, H. Yin, L. Chen, and Y. Zhu, "Ultra-sensitive fluid fill height sensing based on spoof surface plasmon polaritons," Journal of Electromagnetic Waves and Applications, vol. 32, no. 4, pp. 471-482, 2017.

[24] F. Bray, J. Ferlay, I. Soerjomataram, R. L. Siegel, L. A. Torre, and A. Jemal, "Global cancer statistics 2018: GLOBOCAN estimates of incidence and mortality worldwide for 36 cancers in 185 countries," CA: A Cancer Journal for Clinicians, vol. 68, no. 6, p. 394, 2018.

[25] A. Otto, "Excitation of nonradiative surface plasma waves in silver by the method of frustrated total reflection," Zeitschrift für Physik A Hadrons and nuclei, vol. 216, no. 4, pp. 398-410, 1968.

[26] N. Omar, Y. Fen, S. Saleviter et al., "Development of a graphene-based surface plasmon resonance optical sensor chip for potential biomedical application," Materials, vol. 12, no. 12, p. 1928, 2019.

[27] A. Rusina, M. Durach, and I. S. Mark, "Theory of spoof plasmons in real metals," in Proceedings of the Society of Photo-Optical Instrumentation Engineers (SPIE) Conference Series, Austin, TX, USA, June 2010.

[28] X.-F. Zhang, J. Fan, and J.-X. Chen, "High gain and highefficiency millimeter-wave antenna based on spoof surface plasmon polaritons," IEEE Transactions on Antennas and Propagation, vol. 67, no. 1, p. 687, 2019.

[29] Y. Huang, S. Zhong, Y. Shen, L. Yao, Y. Yu, and D. Cui, "Graphene/insulator stack based ultrasensitive terahertz sensor with surface plasmon resonance," IEEE Photonics Journal, vol. 9, no. 6, pp. 1-11, 2017.

[30] S. A. Maier, Plasmonics: Fundamentals and Applications, Springer, Berlin, Germany, 2007.

[31] T. K. Biwas and A. K. Gupta, "Retrieval of true color of the internal organ of CT images and attempt to tissue characterization by refractive index: initial experience," The Indian Journal of Radiology and Imaging, vol. 12, no. 2, pp. 169-178, 2002.

[32] W. M. Nouman, S. E.-S. Abd El-Ghany, S. M. Sallam, A.-F. B. Dawood, and A. H. Aly, "Biophotonic sensor for rapid detection of brain lesions using 1D photonic crystal," Optical and Quantum Electronics, vol. 52, no. 6, p. 287, 2020.

[33] X. Li, J. Song, and J. X. J. Zhang, "Integrated terahertz surface plasmon resonance on polyvinylidene fluoride layer for the profiling of fluid reflectance spectra," Plasmonics, vol. 11, no. 4, pp. 1093-1100, 2015.

[34] M. Chen, L. Singh, N. Xu, R. Singh, W. Zhang, and L. Xie, "Terahertz sensing of highly absorptive water-methanol mixtures with multiple resonances in metamaterials," Optics Express, vol. 25, 2017.

[35] L. Liang, X. Hu, L. Wen et al., "Unity integration of grating slot waveguide and microfluid for terahertz sensing," Laser \& Photonics Reviews, vol. 12, no. 11, p. 1800078, 2018.

[36] X. Zhang, W. Fan, and C. Song, "Multiple plasmonic resonance excitations on graphene metamaterials for ultrasensitive terahertz sensing," Carbon, vol. 133, pp. 416-422, 2018.

[37] X. Chen and W. Fan, "Toroidal metasurfaces integrated with microfluidic for terahertz refractive index sensing," Journal of Physics D: Applied Physics, vol. 52, no. 48, p. 485104, 2019.
[38] F. Chen, Y. Cheng, and H. Luo, "Temperature tunable narrow-band terahertz metasurface absorber based on InSb micro-cylinder arrays for enhanced sensing application," IEEE Access, vol. 8, pp. 82981-82988, 2020. 